\title{
Results of full-scale and laboratory studies of the spillway of HPP No. 2
}

\author{
Ulmas Khusankhudzaev ${ }^{1 *}$, Odil Kadirov ${ }^{2}$, and Azizjon Jakhonov ${ }^{2}$ \\ ${ }^{1}$ Tashkent Institute of Architecture and Civil Engineering, Tashkent, Uzbekistan \\ ${ }^{2}$ Tashkent Institute of Irrigation and Agricultural Mechanization Engineers, Tashkent, Uzbekistan
}

\begin{abstract}
In the article, based on the results of field measurements and calculations, recommendations are given for determining the roughness coefficient of the channel of the spillway. Based on the analysis of the literature and field research data, it was recommended to take the roughness coefficient of the channel of the spillway (canal) equal to $n=0.02$ - for areas with steep slopes and without sediment deposits, and in areas with the worst condition of concrete surfaces and sediment deposits, take $n=0.0225$. According to the results of field surveys of the canal, it was found that the hydraulic regime at the turning section No. 2 in the plan is characterized by an uneven distribution of depths in its crosssections. This is due to the curvilinearity of the turning section of the channel, which has an unacceptably small radius of curvature in the plan causing centrifugal forces and rising levels along the concave wall, which led to an overflow of water over the top of the channel walls. As a result of the conducted experimental - laboratory studies of the turning section of the outflow tract (channel), recommendations are given for reducing the water incursion on the banks, the height of the walls.
\end{abstract}

\section{Introduction}

In recent years, in hydraulic construction, both abroad and the Republic of Uzbekistan, such urgent problems of our time as the observed decrease in the reliability and safety of the operation of hydraulic structures and their elements have come to the fore. The efficiency of water distribution management and the timeliness of water supply to consumers, taking into account the requirements of environmental protection, depend on the stable operation of a hydraulic structure.

Therefore, there is an urgent need for research aimed at the development and implementation of modern technological elements of hydraulic structures, ensuring their trouble-free operation and ensuring their safety requirements. This is especially true for hydroelectric facilities in long-term operation, of the order of 20-40 years or more. Currently, large-scale measures are being taken in the Republic of Uzbekistan to improve the design of hydraulic structures, ensure their reliable and safe operation, improve the throughput and operating mode of spillway structures, and improve their effective mechanisms of work.

*Corresponding author: husan_1954@mail.ru 
The Strategy of Actions for the Further Development of the Republic of Uzbekistan for 2017-2021 outlines tasks, including "development of land reclamation and irrigation facilities, to increase the competitiveness of the national economy." The solution of these tasks, during a period of shortage, including one important task is to carry out research work aimed at developing rational design methods and effective operation, based on accounting for water resources of emerging damages, failures and accidents, as well as stress-deformation processes in the work of hydraulic structures of ground irrigation systems.

In connection with the above, we carried out field and laboratory studies of the outlet duct (channel) of HPP № 2, which showed that the channel does not meet safety requirements.

The channel (channel) outlet section with a total length of $1221.0 \mathrm{~m}$ is concreted, of the trapezoidal cross-section with variable width along the bottom $b=6-27 \mathrm{~m}$ and slope setting coefficients $\mathrm{t}=0-2$. The slope of the channel bottom along the route is variable and varies within $\mathrm{J} 1=0.0248-0.0091$ (in the initial section) $\mathrm{J} 2=0.00725+0$ (in the rest).

The channel is winding in plan and has 8 turning sections, two of which have unacceptable radii of curvature. Also, one railway and two road bridges cross the canal route within its length.

\section{Materials and Methods}

The main questions of the present research were:

- determination of the roughness coefficient of the spillway channel according to the results of field measurements.

-conducting experimental and laboratory studies of the turning section of the outflow tract with the issuance of recommendations for reducing the water incursion on the banks, the height of the walls, etc.

1. Determination of the roughness coefficient of the channel of the spillway according to field survey data

As you know, in design practice, when assigning the roughness coefficient, they mainly use tabular data available in the regulatory literature [2].

However, the actual value of the roughness coefficient may differ from the tabular values (in particular, upward) for some reasons:

- overgrowth with vegetation after a certain period of operation due to wear of the surface of the slopes and the bottom;

- various obstacles on the way of the flow in the channel: bridge abutments, sharp curvatures of the channel, etc.

- the presence of suspended and in the flow, especially bottom pumps:

- depending on the flow rates of water passed through the canal.

At low flow rates and, accordingly, water levels, channel surface roughness, various obstacles in the form of bridge abutments, etc., more noticeable than with high water flows. All of these listed factors are present in the outflow tract (channel) we are examining.

So, on the outlet channel, there are four obstacles: one reinforced concrete, two road bridges, eight turns, and two of them have an unacceptably small radius of curvature, etc.

Therefore, initially adopted when designing the channel, the roughness coefficient is $n=0.016$ naturally subject to adjustment.

To determine the roughness coefficient according to the data of field measurements carried out during the tests of the outlet duct, we will use the following method:

a) a relatively straight section of the canal is selected with an approximately uniform slope, a characteristic bottom and slopes;

b) the marks of water horizons are measured at the beginning and end of the sections: 
d) the average hydraulic parameters of this section are determined at a known flow rate;

e) the slope of the water surface in this area is determined;

f) the speed characteristic $\mathrm{W}^{-}$is found:

$$
\bar{W}=Q /\left(\omega^{-} \sqrt{J}\right)
$$

3) the roughness coefficient " $n$ " is found according to the formula of N.I. Pavlovsky:

$$
n=R^{z} / \bar{W}
$$

where: $\mathrm{R}$ is hydraulic radius of the free area, $\mathrm{m}$;

$$
Z=1 / 2+y
$$

$\mathrm{y}$ is exponent taken equal to $1 / 6$

Substituting these parameters into the formula, we get:

$$
n=R^{(1 / 2+1 / 6)} / \bar{W}=R^{(2 / 3)} / \bar{W}
$$

Such a site was chosen between pop 13 and pop. 14 .

The cross-section of these two sections and their data were taken from the materials of the topographic survey carried out by the maintenance service. The calculation is carried out according to the above method:

transverse -14

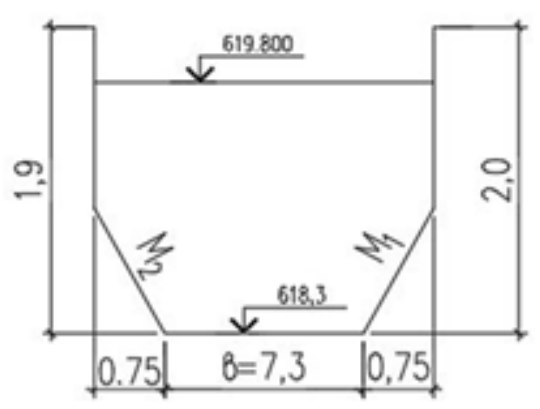

$$
\begin{gathered}
m_{1}=0.263 ; m_{2}=0.250 ; \\
m_{c p}=0.25 ; \\
\omega_{1}=\left(b+m_{c p} h\right) h
\end{gathered}
$$$$
\omega_{1}=(7.3+0.251 .75) 1.75=13.54 \mathrm{~m}^{2} \text {; }
$$

$$
\begin{gathered}
\chi_{l}=\left(b+2 h_{1} \sqrt{1+m_{c p}^{2}}\right)= \\
=7.9+2 \cdot 1.75 \cdot 1.031 \cdot=10.9 \mathrm{~m}
\end{gathered}
$$

transverse -13

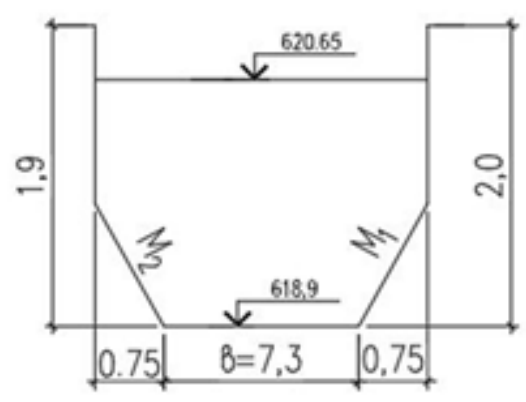

$$
\begin{gathered}
m_{1}{ }^{\prime}=0.3 ; m_{2}^{\prime}=0.316 ; \\
m_{c p}{ }^{\prime}=0.308 ; \\
\Phi_{1}^{\prime}=\left(b+m_{c p} h_{2}\right) h_{2} \\
\Phi_{1}{ }^{\prime}=(7.5+0.316 \cdot 1.5) 1.5=11.96 \mathrm{~m}^{2} ; \\
\chi_{1}^{\prime}=\left(b+2 h_{2} \sqrt{1+m_{c p}^{2 \prime}}\right)= \\
=7.5+2 \cdot 1.5 \cdot 1.046 \cdot=10.64 \mathrm{~m}
\end{gathered}
$$

Determine the average hydraulic elements:

$$
\begin{gathered}
\bar{\omega}=\frac{\omega_{1}+\omega_{2}}{2}=\frac{13.54+11.96}{2}=12.75 \mathrm{~m}^{2} \\
\bar{\chi}=\frac{\chi_{1}+\chi_{2}}{2}=\frac{10.9+10.64}{2}=10.77 \mathrm{~m}
\end{gathered}
$$




$$
\begin{gathered}
\bar{R}=\frac{\bar{\omega}}{\bar{\chi}}=\frac{12.75}{10.77}=1.183 \mathrm{~m} \\
\bar{W}=\frac{Q}{\bar{\omega} \sqrt{J_{2}}} \\
\bar{W}=\frac{30}{12.75 \sqrt{0.0057}}=31.15
\end{gathered}
$$

where: $\bar{W}$ is flow characteristic of the channel

$\mathrm{J}_{2}$ is hydraulic slope of the outflow section $J_{2}=\frac{626.65-1619.8}{156}=0.0057$

Determine the roughness coefficient by the formula (4).

$$
n=\frac{1.183^{2 / 3}}{31.15}=0.035
$$

As calculations show for the accepted section of the canal and the measurements of the hydraulic elements of the section carried out in kind with a water flow rate of $30 \mathrm{~m}^{3} / \mathrm{s}$, the roughness coefficient will be equal to 0.035 , which seems unrealistic.

According to reference and literature data [2-4], the roughness coefficient for channels with concrete lining, even with very poor concrete work and the presence of gravel and sand at the bottom, can be no more than 0.0225 .

If the methodology for calculating the roughness coefficient is beyond doubt, then their direct use in calculations to determine the channel roughness coefficient is unauthorized.

We analyzed the information available in the act drawn up based on the results of passing water flows through the canal, namely:

a) the fact that the water horizons along the canal route were determined by the laths applied on the canal slopes with washable paint indicates that splashes of water horizons were recorded at a peak flow rate in the canal, taking into account the wave of the water surface;

b) water depths measured from the bottom of the concrete channel do not reflect the actual depth because the bottom was everywhere covered with gravel-pebble material up to $40 \mathrm{~cm}$ thick;

c) the slope and cross-section of the channel are constantly changing along the route, and it is impossible to select a section of the channel with a prismatic shape and sufficient length to obtain reliable results of calculating the roughness coefficient;

d) the flow rates through the canal were short-term; the flow contained a significant amount of gravel-pebble material (including boulders) carried out from the damper well, which undoubtedly affected the flow depths.

Therefore, in calculating the throughput of the outflow tract, the roughness coefficient should be taken equal to $n=0.02$ - for areas with large slopes and without sediment deposits and in areas with the worst condition of concrete surfaces and sediments $n=$ 0.0225 .

\section{Results and Discussion}

\subsection{Experimental - laboratory studies of the turning section of the outflow tract}

As the results of full-scale tests of the outlet duct showed, the places of sharp turns, which were made in nature with unacceptably small radii of curvature, turned out to be especially 
unfavorable areas on the spillway. This led to the fact that at the turning sections of the canal when the water flow was passed, local velocities increased, additional pressure losses appeared, and at a flow rate of $\mathrm{Q}=30 \mathrm{~m}^{3} / \mathrm{s}$ on the concave side of the turning section, water overflowed through the top of the canal walls.

Therefore, it was proposed to reconstruct the sections of sharp turns of the outflow tract by increasing the radius of curvature of the turns in the plan and additionally build up concrete walls in these sections.

It is recommended to perform turning sections of channels in plan with a radius $R>5 b$. where: $b$ is the channel width along the water's edge.

Figure 2 shows the design option for redeveloping the turning section, which was investigated on the model.

Investigations of the turning section of the outflow tract were carried out on a spatial fragmentary hydraulic model (Figure 1) at a scale of 1:50 conducted according to the rules of gravitational similarity (according to Froude) [3, 4].
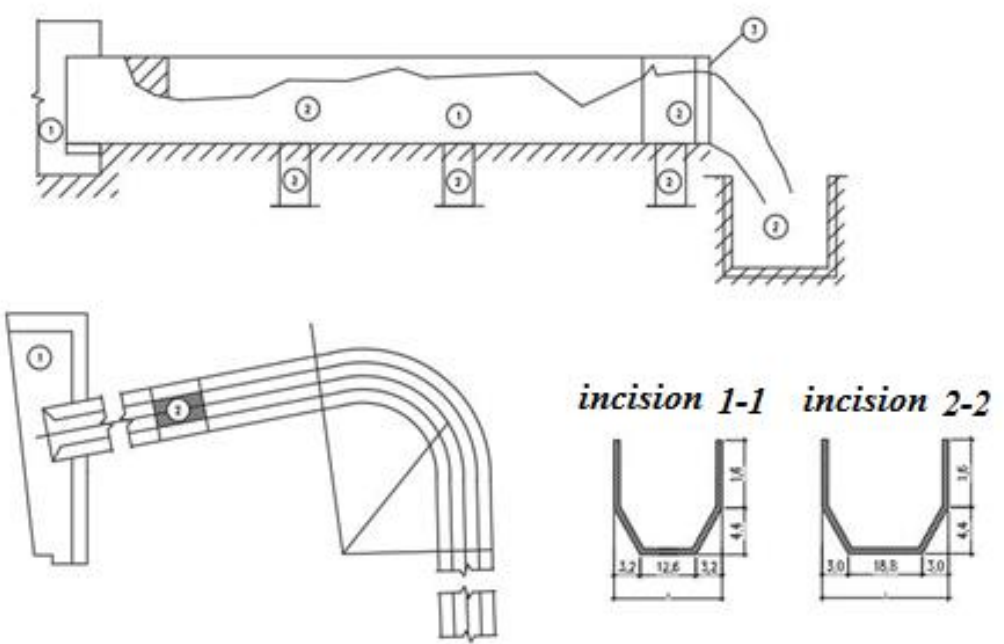

Fig.1. Scheme of the model installation of the turning section No. 2 outflow tract (channel)

Laboratory studies of the design option were carried out at a maximum flow rate $Q=70 \mathrm{~m} 3 / \mathrm{s}$ for two cases:

a) with a slope of the bottom of the approach part $i=0.003$, a slope of the bottom of the turning section $i=0.001$

b) with a slope of the bottom of the approach part $i=0.003$. the slope of the bottom of the turning section $i=0.006$.

The need to conduct experiments for two cases was because in the first case, the elevation marks of the turning section were taken from the available materials of the planned survey, in the second - as a result of repeated topographic survey. Elevation marks were updated and received later. 


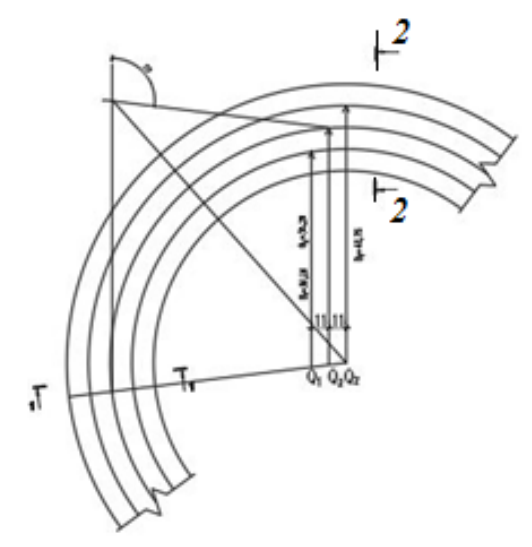

\section{incision 1-1}

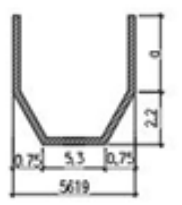

incision 2-2

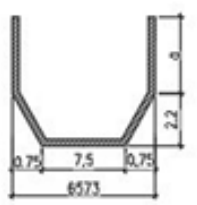

Fig. 2. Recommended diagram of the turning section No. 2 of the outflow tract

As shown by the results of the experiments, the hydraulic regime of the channel on the turning section No. 2 in the plan is characterized by an uneven distribution of depths in its cross-sections. This is due to the curved linearity of the channel section, which has a radial outline in the plan, causing the appearance of centrifugal forces and an increase in levels along the concave wall. In addition, at turn section No. 2, at a certain place, there is a "wave burst", the height of which relative to the channel bottom reached $2.2 \mathrm{~m}$. Moreover, in the first case, the "wave burst" occurred at the beginning of the turning section.

In the second case, in view of the increase in the slope of the channel bottom, the "wave splash" is already observed closer to the end of the turning section.

The height of the channel walls at the turning section should be assigned in accordance with the depths shown in Figure 1, taking into account the margin in the height of the walls.

There is no flow aeration in the investigated section of the turn. Indeed, as is known, flow aeration occurs when the actual Froude number $F r=v^{2} / g h$ exceeds its critical value, which is $[2,5]$.

$$
\begin{gathered}
F_{r}^{k r}=45(1-\Delta / R)^{1 / 4} \\
\mathrm{v}=Q / \omega=Q /(b+m h) h \\
\mathrm{v}=70 /(6.3+0.35 \cdot 1.71) .71=6.94 \mathrm{~m} / \mathrm{s}
\end{gathered}
$$

where: $h$ - average flow depth, m; (the minimum value " $h$ " is set at the beginning of the curve of the turning section in the plan, which is equal to $h=1.71 \mathrm{~m}$ ); $\mathrm{v}$ - average flow velocity $\mathrm{m} / \mathrm{s} ; b$ is channel width at the beginning of the turning section, $b=5.3 \mathrm{~m}$;

$\Delta / R$ is relative surface roughness: for concrete surfaces $\Delta / R=0.05$

Substituting this data into the above formula, we get:

$$
\begin{gathered}
F r=(6.94)^{2} /(9.81 \cdot 1.71)=2.87 \\
F_{r}^{k r}=45(1-0.05) 14=21.9
\end{gathered}
$$

Given that the actual maximum value of the Froude number is less than its critical value, there should be no aeration.

Experimental - laboratory studies to study local erosion behind the end structure of the cantilever spillway to prevent erosion of the bottom and slopes of the outlet channel. 
In more detail, the methodology and results of these studies are presented in [7].

\section{Conclusionss}

1. As the results of field studies and calculations for the spillway (canal) have shown, the roughness coefficient of the bottom and slopes of the canal should be taken equal to $n=0.02$ - for areas with large slopes and without sediment deposits, and in areas with the worst condition of concrete surfaces and sediment deposits take $n=0.0225$.

2. Laboratory studies on the model of the turning section of the channel showed that the hydraulic regime in the channel is characterized by an uneven distribution of depths in its cross-sections. This is due to the curved linearity of the channel section, which has a radial outline in the plan, causing the appearance of centrifugal forces and an increase in levels along the concave wall.

3. The height of the channel walls at the turning section should be assigned in accordance with the depths shown in Figure 1, taking into account the margin in the height of the walls.

(4) In the investigated section of the turn, there is no aeration of the flow since the actual Froude number is less than the critical one $\mathrm{Fr}<\mathrm{F}_{\mathrm{r}}^{k r}, 2.87<21.9$

\section{References}

1. Vizgo M.S. Operational measures and ways to reduce local erosion behind hydraulic structures Ed. Science Uzbekistan Tashkent, p. 218, (1996)

2. Kiselev P.G. Handbook of hydraulic calculations Publishing House Ecolit Moscow (2011)

3. Altunin V.S. Ameliorative channels in earth bed Moskow Publishing House Kolos (1979)

4. Bakiev M.R. Improvement of structures, methods of calculation justification and design of regulatory structures, p. 57, Moscow, (1992)

5. Abdulkarim S.S. Regularities of flow spreading behind transverse dams on rivers with a wide floodplain, p. 27, Moscow, (1991)

6. Abramovich G.N. Theory of turbulent jets. p. 715 Moscow, (1960)

7. Bakiev M.R., Kakhharov U.A., Jakhonov A.A., Panjiev S. Flow parameters in the area compressed by transverse floodplain dams. IOP Conference Series: Materials Science and Engineering, 883(1), 012016 (2020)

8. Bakiev, M.R., Kakhharov, U.A., Jakhonov, A.A., Matkarimov O. Kinematic characteristics of the flow, in the compression region, with bilateral symmetric restriction by floodplain dams. IOP Conference Series: Materials Science and Engineering, 869(7), 072017, (2020)

9. Wormleaten P. R., Merrett D. J. Wormleaton P. R., Merrett D. J. An improved method of calculation for steady uniform flow in prismatic main channel flood plain sections. Journal of hydraulic research. 28,(2), p.157-174, (1990)

10. Knignt D.W., Shiono K. Turbulence measurements in a shear layer region of a compound channel. Journal of hydraulic research, 28,(2), pp.175-196, (1990)

11. Elliot S.C.A., Sellin R.H.J. SERC flood channel facility skewed flow experiments. Journal of hydraulic research, 28,(2), pp.197-214, (1990)

12. Kishanjit K.K., Kanhy Ch.P. Flow distribution in meandering compound channel flow. ISH Journal of Hydraulic Engineering, published online, 07 jun. p. 11-26. http://ascelibrary.org. (2012)

13. Abinash Mohanta, Khatua K.K., Patra K.C. Flow modeling in symmetrically narrowing flood plains. www.siencedirect.com, ICWRCOE, pp.826-833. (2015)

14. Myers W.R., Brennan E.K. Flow resistance in compound channels. Journal of hydraulic research, 28,(2), pp.141-155, (1990) 
15. Mikhalev M.A. Hydraulic calculation of flows with a whirlpool. L.: Energiya p. 184. (1971)

16. Rakhmatullaev, S., Honey, F., Bakiev, M., Metallica-Heino, M., Le Costume, P. Sedimentation of reservoirs in Uzbekistan: A case study of the Akdarya reservoir, Zerafshan River Basin. IAHS-AISH Publication, 349, pp. 171-181, (2011)

17. Khalimbetov A., Bakiyev M., Shukurova S., Choriev Zh., Khayitov K. Studios of submountain river flow patterns constrained by a combined dam. IOP Conference Series: Earth and Environmental Science, 614(1), 012053, (2020)

18. Bakiyev M., Choriev K., J. Field target dimensions of flow constrained by a transverse dam. IOP Conference Series: Materials Science and Engineering, 883(1), 012034, (2020)

19. Khayitov Kh. J. Improving design justification design method for transverse drivers with unilateral floodplain,Tashkent, p. 44 (2018)

20. Zegzhda A.P. Hydraulic losses on friction in channels and pipelines. Moscow, p. 278, (1957)

21. Khasanov Kh. Bakiev M., Choriev J., Jahonov A., Khalimbetov A. Water Reservoir Area and Volume Determination Using Geoinformation Technologies and Remote Sensing. International Journal of Recent Technology and Engineering 4(8) pp. 5458-5461, (2019) 